\title{
ALTERNATIVES TO THE DISCRETE FOURIER TRANSFORM
}

\author{
Doru Balcan, Aliaksei Sandryhaila, Jonathan Gross, Markus Püschel \\ Carnegie Mellon University \\ Pittsburgh, PA 15213
}

\begin{abstract}
It is well-known that the discrete Fourier transform (DFT) of a finite length discrete-time signal samples the discrete-time Fourier transform of the same signal at equidistant points on the unit circle. Hence, as the signal length goes to infinity, the DFT approaches the DTFT. Associated with the DFT are circular convolution and a periodic signal extension. In this paper we identify a large class of alternatives to the DFT using the theory of polynomial algebras. Each of these Fourier transforms approaches the DTFT just as the DFT does, but has its own signal extension and notion of convolution. Further, these Fourier transforms have Vandermonde structure, which enables their fast computation. We provide a few experimental examples that confirm our theoretical results.
\end{abstract}

Index Terms - Discrete Fourier transforms, boundary value problems, spectral analysis, algebra, algebraic signal processing theory, Vandermonde matrix

\section{INTRODUCTION}

The discrete-time Fourier transform (DTFT) for a discrete-time signal with finite support $\mathbf{s}=\left(s_{0}, \ldots, s_{n-1}\right)$ is given by

$$
y(\theta)=\sum_{0 \leq \ell<n} s_{\ell} e^{-j \theta \ell}, \quad \theta \in(-\pi, \pi] .
$$

Computing $y(\theta)$ is equivalent to evaluating the polynomial $s(x)=$ $\sum_{0 \leq \ell<n} s_{\ell} x^{\ell}$ on the unit circle $e^{-j \theta}, \theta \in(-\pi, \pi]$.

For concrete computation usually the discrete Fourier transform (DFT) is used, which computes

$$
y(k)=\sum_{0 \leq \ell<n} s_{\ell} e^{-j \frac{2 \pi k}{n} \ell}, \quad 0 \leq k<n .
$$

Computing $y(k)$ is now equivalent to evaluating $s(x)$ at the $n n$th roots of unity $e^{-2 \pi k j / n}, 0 \leq k<n$ and shows that the DFT in (2) samples the DTFT in (1) at equidistant points on the unit circle. Hence, as $n$ goes to infinity, the DFT approaches the DTFT. Further, it is well-known that applying the DFT assumes that the signal $\mathbf{s}$ is periodically extended and that the associated convolution becomes circular convolution.

Contribution. In this theoretical paper we derive a large set of alternative transforms to the DFT, each of which approaches the DTFT as $n$ goes to infinity. Each of these transforms has its own associated boundary condition and signal extension (which are hence not periodic) and notion of convolution. Further, each of these transforms has a Vandermonde structure, which enables its fast computation using at most $O\left(n \log ^{2}(n)\right)$ operations. We show experimental results with examples of these transforms that confirm our theoretical result and that compare them to the DFT when applied to a signal.

This work was supported by NSF through award 0634967.
The derivation of the alternatives to the DFT makes use of the Beraha-Kahane-Weiss theorem [1] that describes the asymptotic behavior of root sets of polynomials. We combine this theorem with the theory of polynomial algebras [2], which is known to describe the DFT [3] algebraically. This connection was recently extended to the algebraic signal processing theory [4].

Organization. Section 2 explains the polynomial algebra framework underlying both the DFT and the alternative transforms that we derive in this paper. This framework reduces the problem of deriving the alternative transforms to finding sequences of polynomials whose root sets converge to the unit circle. We identify a large class of such sequences in Section 3 and consider a few concrete examples for experiments in Section 4. We conclude with Section 5.

\section{BACKGROUND}

The key to deriving alternatives to the DFT is its interpretation in the framework of polynomial algebras $\mathbb{C}[x] / p_{n}(x)$, which we overview in this section. Every polynomial algebra has an associated notion of boundary condition, signal extension, convolution, spectrum, and Fourier transform, as explained in the algebraic signal processing theory $[5,4]$. As running example, we use $\mathbb{C}[x] /\left(x^{n}-1\right)$, which is known to be associated with the DFT [3].

In short, we will show in this paper that polynomials $p_{n}(x)$ other than $x^{n}-1$ can be used to define alternatives to the DFT.

Polynomial algebra. An algebra is a vector space that is also a ring, i.e., permits the multiplication of its elements. Examples include the complex numbers $\mathbb{C}$ and the complex polynomials $\mathbb{C}[x]$.

Let $p_{n}(x)=x^{n}+\sum_{0<i<n} \beta_{i} x^{i}$ be a (normalized) polynomial of degree $\operatorname{deg}(p)=n$. The set of all polynomials of degree less than $n$,

$$
\mathbb{C}[x] / p_{n}(x)=\left\{s(x)=\sum_{0 \leq \ell<n} s_{\ell} x^{\ell} \mid \operatorname{deg}(s)<n\right\}
$$

with addition and multiplication modulo $p(x)$ is called a polynomial algebra. As a vector space, $\mathbb{C}[x] / p(x)$ has dimension $n$. As a basis, we choose $b=\left(1, x, \ldots, x^{n-1}\right)$. For $s(x) \in \mathbb{C}[x] / p(x)$, we denote the list of coefficients with $\mathbf{s}=\left(s_{0}, \ldots, s_{n-1}\right)$.

As an example, $\mathbb{C}[x] /\left(x^{n}-1\right)$ is a polynomial algebra.

Boundary condition and signal extension. Every $\mathbb{C}[x] / p_{n}(x)$ has an associated (right) boundary condition which is obtained by reducing $x^{n} \bmod p_{n}(x)=-\sum_{0<i<n} \beta_{i} x^{i}$. Similarly, the (right) signal extension is given by reducing $x^{m} \bmod p_{n}(x)$ for $m \geq n$.

In our example, $p_{n}(x)=x^{n}-1$, i.e., $x^{n} \bmod x^{n}-1=1$ is the cyclic boundary condition. Further, $x^{m} \bmod x^{n}-1=x^{m \bmod n}$, i.e., a periodic signal extension.

Convolution. The convolution associated with $\mathbb{C}[x] / p_{n}(x)$ is the multiplication $h(x) s(x) \bmod p(x)$.

In our example $h(x) s(x) \bmod x^{n}-1$ is equivalent to the circular convolution of the coordinate sequences $\mathbf{h}$ and $\mathbf{s}$ [3].

Spectrum and Fourier transform. We assume $p_{n}(x)$ has pairwise distinct zeros $\alpha=\left(\alpha_{0}, \ldots, \alpha_{n-1}\right)$. Then the Fourier transform associated with $\mathbb{C}[x] / p_{n}(x)$ is given by the Chinese remainder 


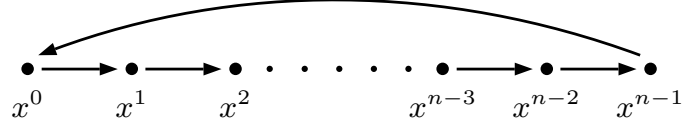

Fig. 1. The structure imposed on the signal by the polynomial algebra $\mathbb{C}[x] /\left(x^{n}-1\right)$ and hence by the DFT.

theorem [2], which decomposes it into a Cartesian product of onedimensional polynomial algebras:

$$
\begin{aligned}
\mathcal{F}: \mathbb{C}[x] / p_{n}(x) & \rightarrow \bigoplus_{0 \leq k<n} \mathbb{C}[x] /\left(x-\alpha_{k}\right), \\
s(x) & \mapsto\left(s\left(\alpha_{0}\right), \ldots, s\left(\alpha_{n-1}\right)\right) .
\end{aligned}
$$

$\mathcal{F}$ is a linear mapping (even an isomorphism of algebras). Hence, with respect to the basis $b$ of $\mathbb{C}[x] / p_{n}(x)$ and $\left(x^{0}\right)=(1)$ in each of the $\mathbb{C}[x] /\left(x-\alpha_{k}\right)$ it is represented by a matrix which takes the form of a Vandermonde matrix (evaluating all basis elements in $b$ at all zeros in $\alpha$ ):

$$
\mathcal{F}=\left[\alpha_{k}^{\ell}\right]_{0 \leq k, \ell<n} .
$$

Note that this class of transforms does not contain the discrete cosine and sine transforms, which can be captured in the algebraic framework by using Chebyshev polynomials $[6,4]$.

In our example, the zeros of $x^{n}-1$ are $\alpha_{k}=\omega_{n}^{k}, \omega_{n}=$ $\exp (-2 \pi j / n)$. Hence $\mathcal{F}=\left[\omega_{n}^{k \ell}\right]_{0 \leq k, \ell<n}=\mathrm{DFT}_{n}$ is exactly the discrete Fourier transform, i.e., the $y_{k}$ in (2) are computed as $\mathbf{y}=\mathcal{F} \mathbf{s}, \mathbf{y}=\left(y_{0}, \ldots, y_{n-1}\right)$.

Visualization. The operation of $x$ on the basis $b$ of $\mathbb{C}[x] / p_{n}(x)$ can be represented by a graph. E.g., in our example $p_{n}(x)=x^{n}-1$, we obtain the directed circle in Fig. 1. Note how the graph captures the boundary condition $x^{n}=x^{0}$. Intuitively, the graph is the structure imposed on a signal by the polynomial algebra.

Fast algorithms. Every general Fourier transform in (4) is a Vandermonde matrix and hence possesses algorithms with $O\left(n \log ^{2}(n)\right.$ runtime [7]. In the case of the DFT, even $O(n \log (n))$ is possible.

\section{ALTERNATIVE DISCRETE FOURIER TRANSFORMS}

Problem statement. We are interested in finding polynomial algebras $\mathbb{C}[x] / p_{n}(x)$ such that the set of zeros of $p_{n}$ converges to the unit circle as $n$ goes to infinity. The theory in Section 2 yields for each choice of $p_{n}(x)$ the associated notions of signal extension, convolution, spectrum, and Fourier transform. By construction, the latter will approach the DTFT in (1) as $n$ goes to infinity, just as the DFT (which arises from the special case $p_{n}(x)=x^{n}-1$ ) in (2) does.

We will use the following definition.

Definition 1 Let $\left\{p_{n}(x) \mid n \geq 0\right\}$ be a sequence of complex polynomials of increasing degree $\operatorname{deg}\left(p_{n}\right)=n$. We say that $z \in \mathbb{C}$ is a limit of zeros for this sequence if there is a sequence $\left\{z_{n} \mid n \geq 0\right\}$ such that $p_{n}\left(z_{n}\right)=0$ and $\lim _{n \rightarrow \infty} z_{n}=x$.

As an example, the limits of zeros of the sequence given by $p_{n}(x)=x^{n}-1$ are precisely all points on the unit circle. Let us observe that we can extend the definition above for families $\left\{q_{n}(x)\right\}$ of polynomials with increasing degrees (not necessarily equal to their index).

Main theorem. The main result of this paper is the following theorem, which yields a large class of sequences of polynomials whose zero sets converge to the unit circle. We determine and experimentally test the associated alternatives to the DFT later.
Theorem 1 Let

$q_{n}(x)=a_{k}(x) x^{k n}+a_{k-1}(x) x^{(k-1) n}+\ldots+a_{1}(x) x^{n}+a_{0}(x)$,

where $a_{i}(x) \in \mathbb{C}[x]$ and $a_{0}, a_{k} \neq 0$. Then, $z \in \mathbb{C}$ is a limit of zeros if and only if one of the following holds:

$$
\begin{aligned}
& \text { i. }|z|=1 . \\
& \text { ii. }|z|<1 \text { and } a_{0}(z)=0 . \\
& \text { iii. }|z|>1 \text { and } a_{k}(z)=0 .
\end{aligned}
$$

In other words, Theorem 1 states that the limits of zeros of the polynomial sequence in (5) is the entire unit circle, plus possibly finitely many additional points, namely the roots of $a_{0}(x)$ inside the unit circle and the roots of $a_{k}(z)$ outside the unit circle.

This result can be readily extended by combining such families of polynomials, which yields the following corollary.

Corollary 1 Let $p_{n}(x)=\sum_{i=0}^{k} a_{i}(x) x^{\left\lfloor\frac{i(n-d)}{k}\right\rfloor}$ with $a_{i}(x) \in \mathbb{C}[x]$ and $a_{0}, a_{k} \neq 0, d=\operatorname{deg}\left(a_{k}\right)$. Then $z \in \mathbb{C}$ is a limit of zeros for this sequence if and only if one of i.-iii. in Theorem 5 holds.

$p_{n}(x)=x^{n}-1$ is a special case of the sequence in Corollary 1.

To prove Theorem 1, we use a theorem from Beraha, Kahane, and Weiss [1] explained next.

The Beraha-Kahane-Weiss theorem. Suppose $\left\{q_{n} \mid n \geq 0\right\}$ is a sequence of polynomials satisfying the $m$-th degree recursion

$$
q_{n+m}(x)=-\sum_{j=1}^{m} f_{j}(x) q_{n+m-j}(x),
$$

where the $f_{j} \in \mathbb{C}[x]$ are polynomials. For each $x \in \mathbb{C}$, (6) is an ordinary linear recurrence for the numbers $q_{n}(x), n \geq 0$. With this observation, we can solve (6) following the standard procedure for linear recurrences [8, app.A], except that the results depend on $x$.

The characteristic equation associated with (6) is

$$
Q_{x}(\lambda)=\lambda^{m}+\sum_{j=1}^{m} f_{j}(x) \lambda^{m-j}=0 .
$$

Let $\lambda_{1}(x), \ldots, \lambda_{m}(x)$ be the $m$ zeros of of $Q_{x}$. If the $\lambda_{j}(x)$ are pairwise distinct for a particular $x$, then $q_{n}(x)$ has the form

$$
q_{n}(x)=\sum_{j=1}^{m} \alpha_{j}(x) \lambda_{j}(x)^{n},
$$

where the $\alpha_{j}$ are determined by solving a system of $m$ linear equations obtained by letting $n=0,1, \ldots, m-1$. If the $\lambda_{j}(x)$ are not pairwise distinct, (8) is adjusted in the usual way [8, app.A].

We assume that the following two nondegeneracy conditions are satisfied:

- $\left\{q_{n}\right\}$ does not satisfy a recursion of degree less than $m$.

- There are no $i, j$ such that $\lambda_{i}(x) \equiv \omega \lambda_{j}(x)$ for a constant $\omega$ with $|\omega|=1$.

Under these conditions, the following theorem holds.

Theorem 2 A point $z \in \mathbb{C}$ is a limit of zeros of $\left\{q_{n}\right\}$ if and only if the $\lambda_{j}(z)$ can be ordered such that one of the following holds:

(i). $\left|\lambda_{1}(z)\right|>\left|\lambda_{j}(z)\right|, 2 \leq j \leq m$, and $\alpha_{1}(z)=0$ 
(ii). $\left|\lambda_{1}(z)\right|=\left|\lambda_{2}(z)\right|=\ldots=\left|\lambda_{l}(z)\right|>\left|\lambda_{j}(z)\right|, l+1 \leq j \leq$ $m$, for some $l \geq 2$.

Proof of Theorem 1. To apply Theorem 2, we show that our polynomial sequence (5)

- satisfies a linear recursion,

- allows for a simple computation of the roots $\lambda_{j}(x)$ and of the coefficients $\alpha_{j}(x)$ in (8), and

- satisfies the nondegeneracy conditions.

Lemma 1 Let $\left\{q_{n}\right\}$ be the sequence defined in (5) and let $I=\{i \mid$ $\left.0 \leq i \leq k, a_{i}(x) \neq 0\right\} \equiv\left(0=i_{1}<i_{2}<\ldots<i_{m}=k\right)$. Then $\left\{q_{n}\right\}$ satisfies the following recurrence of order $m=|I|$, and no recurrence of smaller order:

$$
q_{n}(x)=-\sum_{j=1}^{m} f_{j}(x) q_{n-j}(x),
$$

where the polynomials $f_{j}$ are defined as

$$
f_{j}(x)=(-1)^{j} \sum_{J \subset I,|J|=j} \prod_{\ell \in J} x^{\ell} .
$$

Further, the characteristic equation takes the simple form

$$
Q_{x}(\lambda)=\lambda^{m}+\sum_{j=1}^{m} f_{j}(x) \lambda^{m-j}=\prod_{i \in I}\left(\lambda-x^{i}\right),
$$

which implies $\lambda_{j}(x)=x^{i_{j}}$; hence the nondegeneracy condition is satisfied. Comparing (8) with (5), this also shows $\alpha_{j}(x)=a_{i_{j}}(x)$.

In particular, the recurrence for the $q_{n}$ does not depend on the $a_{i}(x)$ in (5); the $a_{i}$ will affect only the initial conditions.

Proof. First we prove that $\left\{q_{n}(x)\right\}$ indeed satisfies the relation above by induction on $|I|$. If $m=1$ (implying $k=0$ ) the statement holds, since $q_{n}(x)=a_{0}(x)=q_{n-1}(x), f_{1}(x)=-1$, and $Q_{x}(\lambda)=\lambda-1$.

Suppose now that the statement is true for $m-1 \geq 1$. We shall prove it also holds for $m$ (implicitly, $m \geq 2$ and therefore $k>0$ ). Let $I_{k}=I \backslash\{k\} \neq \emptyset$ (i.e., $I$ without its maximum element) and

$$
r_{n-1}(x)=q_{n}(x)-x^{k} q_{n-1}(x)=\sum_{i \in I_{k}} b_{i}(x) x^{i(n-1)},
$$

where for all $i \in I_{k}, b_{i}(x)=a_{i}(x)\left(x^{i}-x^{k}\right) \neq 0$. Define $\widetilde{f}_{j}(x)=$ $(-1)^{j} \sum_{J \subset I_{k},|J|=j} \prod_{\ell \in J} x^{\ell}$, where $1 \leq j \leq m-1$. As $\left|I_{k}\right|=m-1$, by applying the induction hypothesis to the sequence $\left\{r_{n}\right\}$, we find

$$
\begin{aligned}
r_{n-1}(x)= & -\sum_{j=1}^{m-1} \tilde{f}_{j}(x) r_{n-1-j}(x) \\
= & -\widetilde{f}_{1}(x) q_{n-1}(x)-\sum_{j=2}^{m-1}\left(\widetilde{f}_{j}(x)-\widetilde{f}_{j-1}(x) x^{k}\right) q_{n-j}(x) \\
& +\widetilde{f}_{m-1}(x) x^{k} q_{n-m}(x) .
\end{aligned}
$$

We conclude the proof of the first claim in our lemma by observing that $-\widetilde{f}_{1}(x)=-f_{1}(x)-x^{k}, \widetilde{f}_{m-1}(x) x^{k}=-f_{m}(x)$, and $-\widetilde{f}_{j}(x)+\widetilde{f}_{j-1}(x) x^{k}=-f_{j}(x)$ for $1<j<m$, which implies

$$
q_{n}(x)=r_{n-1}(x)+x^{k} q_{n-1}(x)=-\sum_{j=1}^{m} f_{j}(x) q_{n-j}(x) .
$$

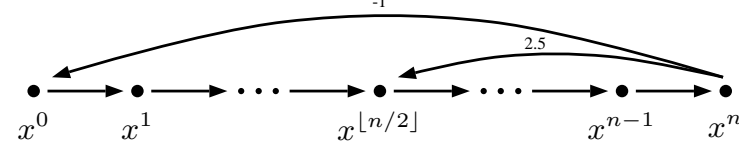

Fig. 2. The structure imposed on the signal by $\mathbb{C}[x] /\left(x^{n}-\right.$ $\left.\frac{5}{2} x^{\lfloor n / 2\rfloor}+1\right)$ and its associated Fourier transform.

To show that $\left\{q_{n}\right\}$ does not satisfy any recursion of order smaller than $m$, we use proof by contradiction but omit the details due to space limitations.

At this point we have shown that Theorem 2 is applicable to (5). To complete the proof of Theorem 1 we inspect which points satisfy one of the two conditions in Theorem 2 . If for a $z \in \mathbb{C}$, exactly one of $\left|\lambda_{j}(z)\right|=\left|z^{i_{j}}\right|$ is maximal, then $|z| \neq 1$. In the case $|z|>1$, we know $\left|z^{k}\right|>\left|z^{i}\right|$, for $i \in I \backslash\{k\}$, and so $z$ is a limit of zeros for $\left\{q_{n}\right\}$ if and only if $a_{k}(z)=0$. In the case $|z|<1$, we have $1=\left|z^{0}\right|>\left|z^{i}\right|$, for $i \in I \backslash\{0\}$ and $z$ is a limit of zeros if and only if $a_{0}(z)=0$. This completely handles the first condition in Theorem 2. Alternatively, if for $z \in \mathbb{C}$, there are $i, j \in I, i \neq j$, such that $\left|z^{i}\right|=\left|z^{j}\right|$, then necessarily $|z|=1$. Since for all $z$ on the unit circle $1=\left|z^{i}\right|, i \in I$, we conclude that any such point is a limit of zeros for $\left\{q_{n}\right\}$. This completes the proof of Theorem 1 .

Associated Fourier transforms. For each polynomial sequence $p_{n}$ of the form considered above, and hence polynomial algebra $\mathbb{C}[x] / p_{n}$, the general theory from Section 2 provides the associated notions of boundary condition and signal extension (which will not be periodic in general), convolution, spectrum, and Fourier transform. The latter will be an alternative to the DFT, and has a fast algorithm due to its Vandermonde structure (Section 2).

\section{EXAMPLE AND EXPERIMENTS}

Example. As a first example, we consider the polynomials $p_{n}(x)=$ $x^{n}-\frac{5}{2} x^{\lfloor n / 2\rfloor}+1$, which match Corollary 1 , and apply the theory in Section 2.

The boundary condition in $\mathbb{C}[x] / p_{n}$ is given by $x^{n}=\frac{5}{2} x^{\lfloor n / 2\rfloor}$ 1 , which yields the visualization in Fig. 2. Convolution is the multiplication of polynomials $h(x) s(x) \bmod p_{n}(x)$. The Fourier transform $\mathcal{F}$ in (4) is determined by the zeros of $p_{n}$. For even $n=2 m$ they can be explicitly computed as

$$
\alpha=\left(\sqrt[m]{2} w_{m}^{-m / 2+k}, \frac{1}{\sqrt[m]{2}} w_{m}^{-m / 2+k}\right)_{1 \leq k \leq m},
$$

We order the roots by their angles from $-\pi$ to $\pi$. The roots distribution for $n=20$ and 80 is shown in Fig. 4(b) below.

Hence the Fourier transform in the case $n=2 m$ becomes

$$
\mathcal{F}_{2 m}=\left[2^{\frac{(-1)^{k} l}{m}} w_{m}^{\left(\left\lfloor\frac{k}{2}\right\rfloor+1-\frac{m}{2}\right) l}\right]_{0 \leq k, l<2 m} .
$$

Experiments. For our experiments, we consider four sequences of polynomials; the first is associated with the DFT:

$$
\begin{aligned}
& p_{n}(x)=x^{n}-1, \\
& p_{n}(x)=x^{n}-\frac{5}{2} x^{\lfloor n / 2\rfloor}+1, \\
& p_{n}(x)=\left(4 x^{3}+1\right) x^{n-3}+\left(5 x^{2}+1\right) x^{\left\lfloor\frac{n-3}{2}\right\rfloor}+\left(7 x^{5}+1\right), \\
& p_{n}(x)=\left(2 x^{3}+3\right) x^{n-3}-\left(x^{5}-2\right) .
\end{aligned}
$$

In each case, we numerically compute the root set of $p_{n}(x)$ for $n \in\{20,50,80\}$, construct the corresponding Fourier transform, 


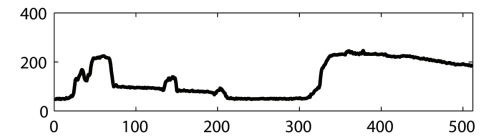

Fig. 3. Sample signal.
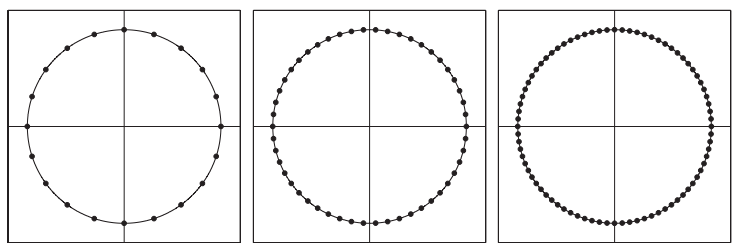

(a) $p_{n}(x)=x^{n}-1$
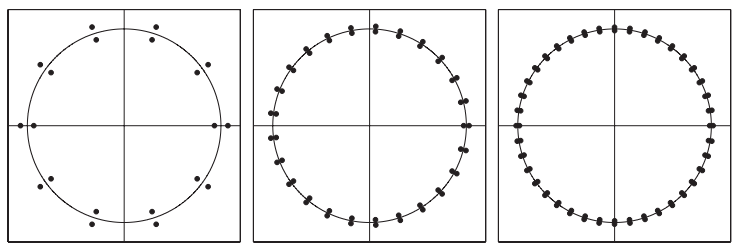

(b) $p_{n}(x)=x^{n}-\frac{5}{2} x^{\left\lfloor\frac{n}{2}\right\rfloor}+1$
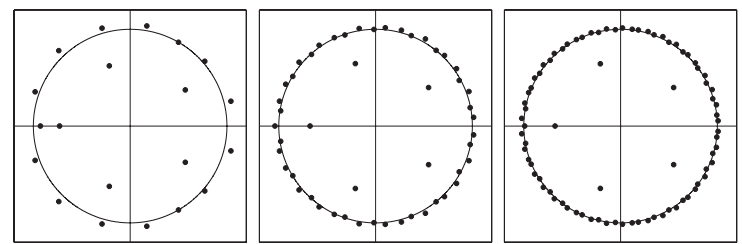

(c) $p_{n}(x)=\left(4 x^{3}+1\right) x^{n-3}+\left(5 x^{2}+1\right) x^{\left\lfloor\frac{n-3}{2}\right\rfloor}+\left(7 x^{5}+1\right)$
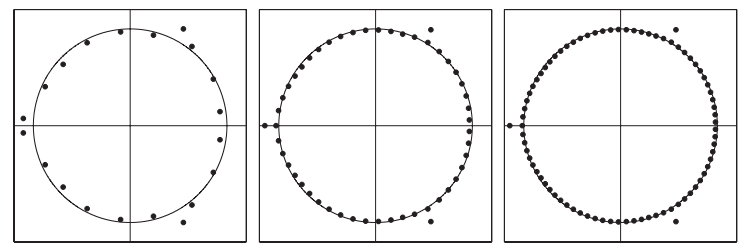

(d) $p_{n}(x)=\left(2 x^{3}+3\right) x^{n-3}-\left(x^{5}-2\right)$

Fig. 4. Roots of polynomials $p_{n}(x)$ for $n=20,50$, and 80 .

and apply it to the first $n$ coefficients of the sample signal shown in Fig. 3, which is one row of a gray-scale image.

According to Theorem 1, all roots of the polynomial sequences (15) and (16) converge to the unit circle. The sequence (17) has five limits of zeros inside the unit circle: $z_{k}=\sqrt[5]{1 / 7} e^{\pi i(2 k+1) / 5}$, $0 \leq k \leq 4$. The sequence (18) has three limits of zeros outside the unit circle: $z_{k}=\sqrt[3]{3 / 2} e^{\pi i(2 k+1) / 3}, 0 \leq k \leq 2$. This is confirmed by Fig. 4, which shows the root sets for $n \in\{20,50,80\}$.

Fig. 5(a)-5(b) shows, for $n \in\{20,80\}$, the associated Fourier transform applied to the signal in Fig. 3. We observe that the spectra become similar for $n=80$ as expected. In the last case, the three limits of zeros outside the unit circle cause the three associated spectral values be unbounded with respect to $n$. In contrast, the five limits of zeros inside the unit circle in the third case do not cause this behaviour.

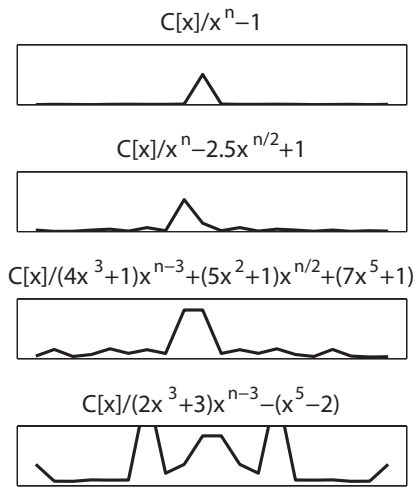

(a) $n=20$

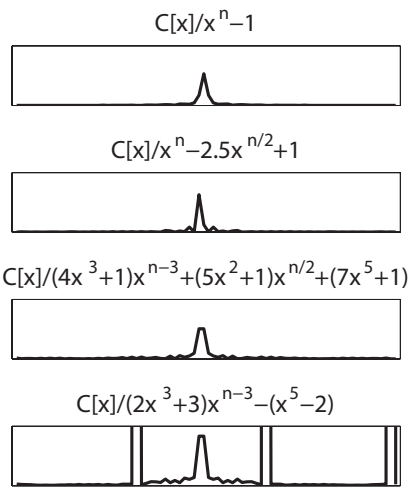

(b) $n=80$
Fig. 5. Fourier transform of the sample signal for $\mathbb{C}[x] / p_{n}(x)$ for $n=20,80$.

\section{CONCLUSION}

The question we addressed in this paper is arguably fundamental to signal processing: why do we use a periodic signal extension and hence a DFT for finite length discrete-time signals? We showed that if only asymptotic convergence to the DTFT is required, there are indeed many choices, each of which with its own signal extension and notion of convolution. Further, each of these alternative transforms possesses fast algorithms, which makes them in principle useful for applications. The question of these applications still remains. One source may be those, such as image processing, in which the assumption of periodicity is unrealistic. For those, our class of transforms may prove useful.

\section{REFERENCES}

[1] S. Beraha, J. Kahane, and N. J. Weiss, "Limits of zeros of recursively defined families of polynomials," in Studies in Foundations and Combinatorics: Advances in Math., Supplementary Studies, vol. I, pp. 213-232. Academic Press, 1978.

[2] Paul A. Fuhrman, A Polynomial Approach to Linear Algebra, Springer Verlag, 1996.

[3] H. J. Nussbaumer, Fast Fourier Transformation and Convolution Algorithms, Springer, 2nd edition, 1982.

[4] M. Püschel and J. M. F. Moura, "Algebraic signal processing theory," available at http://arxiv.org/abs/cs.IT/0612077, parts of this manuscript are submitted as [5].

[5] M. Püschel and J. M. F. Moura, "Algebraic signal processing theory: Foundation and 1-D time," submitted for publication, part of [4]

[6] M. Püschel and J. M. F. Moura, "The algebraic approach to the discrete cosine and sine transforms and their fast algorithms," SIAM Journal of Computing, vol. 32, no. 5, pp. 1280-1316, 2003.

[7] V. Olshevsky and A. Shokrollahi, "Fast matrix-vector multiplication algorithms for confluent Cauchy-like matrices with applications," in Proc. of the 32nd ACM Symposium on Theory of Computing (STOC'00), New York, 2000, pp. 573-581, ACM.

[8] S. Lipschutz and M. Lipson, Discrete Mathematics, McGraw Hill, 2 edition, 1997. 